\title{
A Method about Printing Special Characters Using the Micro Printer
}

\author{
Wei-Dong ZHANG ${ }^{1, a}$ \\ ${ }^{1}$ Air Force Aviation University, Chang Chun, China \\ azwd1970@163.com
}

Keywords: Single Chip Microcomputer, The Micro Printer, Special Character.

\begin{abstract}
This paper introduced the capability and characteristic of micro-printer, and method of single chip microcomputer connecting with micro-printer. It's important to illuminates the ideas and concrete steps to achieve how micro-printer is controlled to print special character by single chip microcomputer.

The micro printer with its characteristics such as compact, reliable, is widely used in various fields. They not only can print the recording data constantly, but also can print special characters out of character stock by control. The general characters warehouse in a micro printer only consist international first and second class of Chinese characters. So printing Chinese characters or special characters can not be in progress by the order of a micro printer for Chinese characters. This paper mainly discuss the connection of a single-chip microcomputer and a micro printer the way to print special characters, only describe focus on the realizing progress, the specific program is omitted.
\end{abstract}

\section{The Principal Technical Function of a Micro Printer}

At present, a micro printer is used widely, it has many varieties, for example the micro printer series of TP, UP and MP-T, etc. The principal technical functions are introduced as below:

1) It has super micro volume and super power consumption, the size general is $160 \mathrm{~mm}$ (length) $\times 106 \mathrm{~mm}$ (width) $\times 40 \mathrm{~mm}$ (high). Its current is $6 \mathrm{~mA}$ on standby. Its current source only needs direct-current $5 \mathrm{~V} \pm 0.25 \mathrm{~V}, 1.0 \mathrm{~A}$.

2) Printing concentration can be adjusted. The user can select the printing color level through specialized controlling order.

3) Words processing ability is powerful. It can mix to print the lattice ASCII characters for $16 \times 16,12 \times 12,8 \times 16,16 \times 8,8 \times 8,6 \times 12$, Chinese characters and chart characters.

4) Chinese characters printing function is convenient. Chinese characters micro printer brings its own lattice $16 \times 16$ and lattice $12 \times 12$ international first and second Chinese characters warehouse besides its inner storage of first and second character collection. It can get the inner code of Chinese characters standard printer on tap (or international code) or input Chinese characters directly.

5) The dot and line number of characters on each line can be changed by order, that is the distance of dots and lines between characters of 0 to 256 can be selected at random.

6) There are two ways of interface for parallel and USB. The parallel interface is compatible with the standard CENTRONICS interface. The USB interface is compatible with the standard RS-232 interface. The two interfaces are connected by the standard chip 25, type D plug and cable with the micro printer.

7) The reliability is high. Chinese characters micro printer uses import original printing magnetic head. The modularity design of printing head driving circuit can protect the device head, but can last the using life span of heads and ribbons effectively. The reliable resetting controlling circuits, considerate anti-opposite inserting circuits and perfect software and hardware dog-watching controlling circuits can avoid the phenomenon of not working and in a printing mess, can also strengthen the reliability of the overall printer evidently.

8) The compatibility is very good. Generally printing control order of Chinese characters micro printer (except new added control order) and hardware are compatible with common micro printer of different factories completely. This feature can make a printer be multi-used, and it can also be selected on printing speed, printing width and printing type-faces. This also makes users be convenient with the products development or products improvement. 


\section{Using a Single Chip Microcomputer to Drive a Micro Printer}

Take MP-T as an example below to illustrate how a micro-printer accepts the control of a single ship microcomputer.

1) A micro-printer interface for MP-T series.

Chinese characters micro-printers for MP-T series parallel interfaces use needle-like chip D25 socket to be compatible with standard parallel interface CENTRONICS. Those can be controlled directly by micro-printers parallel interfaces or single chips microcomputer.

2) The connection of a MP-T micro-printer and a single chip microcomputer Take PIC16F877A as an example for a single ship. This type of single chip microcomputer belongs to medium-class products of the 8th place, its pin has 40.there are 5 interfaces among them RA0-RA5 are bidirectional interfaces of 6 pins, RB0-RB7 are bidirectional interfaces I/O of the 8th place. Each pin inside adds weak up-pulled electric resistance that can make program, RC0-RC7 also are bidirectional interfaces I/O of the 8th place.RD0-RD7 also are bidirectional interfaces I/O of the 8th place. Meanwhile, RE0-RE2 are bidirectional interfaces I/O of the 8th place.

MP-T micro-printer has lock and storage device in input circuits, it has three - conditional door control. A single chip microcomputer can directly be connected with MP-T min-printer. The connection figure of PIC 16F877A with micro-printer as below: Figure 1

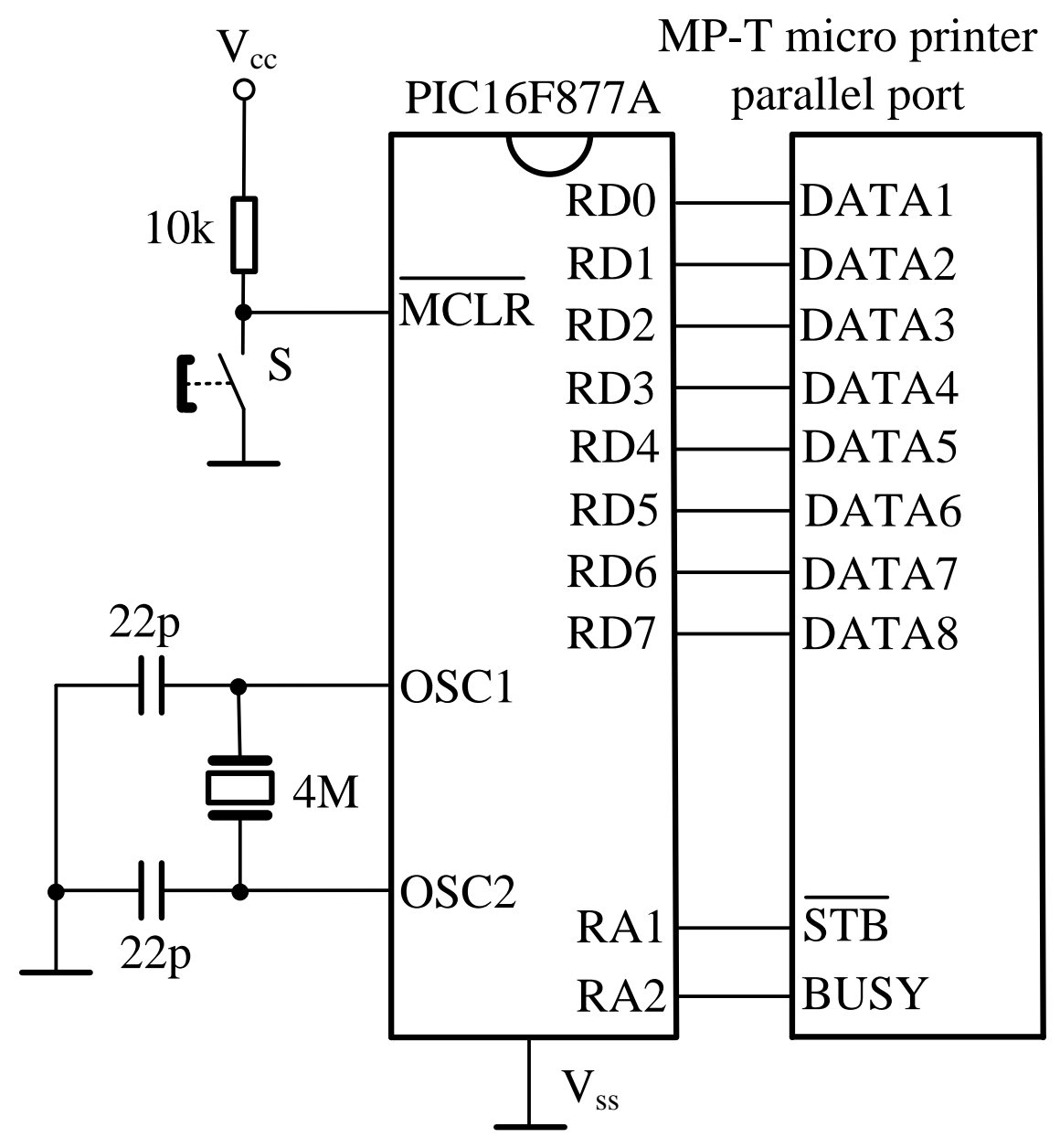

Fig.1 Connection

3) A micro-printer realizes to print by control.

When PIC single chip is connected with a mini-printer, 4 signals in pin are primarily used, selected signals of DATA(data signal DB0-DB7), STB(data communication), BUSY(busy signal), ACK(reply signal).

The logic relationship between the 4 signals is below in Figure 2 


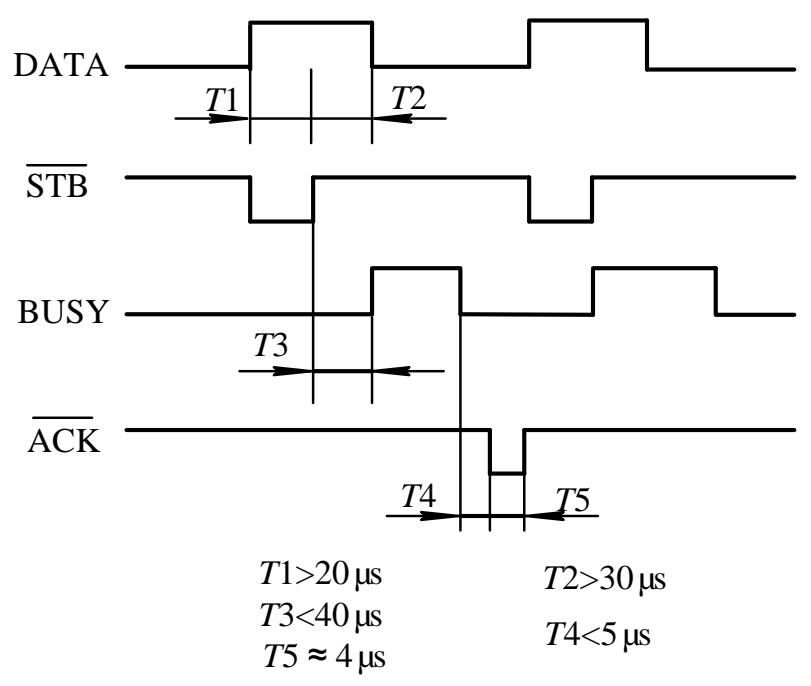

Fig.2 Temporal relations

Its working principals: At first a single chip inquiries if RA2, that is BUSY, is 1 , if BUSY $=0$, it tells that printer isn't busy. It can print. PIC single chip microcomputer outputs data to the printer's data interface DATA, DATA8 through the interface R.D when the data needed to print appear on the data line, RA, that is STB, begins to print with negative impulse rising following time.

A micro-printer generally supplies more than 50 various of printing orders. These orders are composed of a controlling code series. For example, a series of micro-printer MP-T Chinese characters as ASCII code indicating wag or hexadecimal code indicating ways : (1)ASCII code is ESC $8 n$; (2) the hexadecimal code is $1 B 38 n$. $n$ indicates characters printing of different lattice, when $\mathrm{n}=0,16 \times 16$ lattice Chinese characters printing are selected. $\mathrm{N}=1,8 \times 16$ lattice Chinese characters printing is selected. ESC 8 or 1B 38 is control code, after micro-printers receives the order. It starts to work. On Chinese characters printing way, the code inner printer of two bytes is received. A Chinese character lattice code printing in international print or second Chinese characters warehouse can be set up. Working process can find Chinese characters inner code according to Chinese printing order, they make Chinese characters lattice code $(16 \times 16$ lattice code) as lateral sixteen dots, vertical sixteen dots, then the corresponding dots to print Chinese characters are sent out.

\section{Design of Software and Hardware to Print Special Characters}

Printing special characters can't be carried out accordance with Chinese characters printing order. For a micro-printer doesn't have special characters lattice code, but software and hardware can be designed accordance with the basic principal of Chinese characters printing to realize special characters printing.

For Chinese characters order using micro-printer only can print Chinese characters of international first and second class warehouse. Chinese characters printing order makes inner code change into lattice code, makes binary of $16 \times 16$ Chinese characters lattice composed of character chart lattice transport to the printer to print Chinese characters. According to the thinking above, when special characters are printed, a chart printing order can be used. Using chart printing orders define chart lattice. Any special characters can be defined or printed out flexibly. The width of Chinese character isn't restricted with the maximum numbers. AS MP-T series micro-printing pattern printing has two kinds: (1) ASCII code is ESC K n1 n2 data; (2)hexadecimal is : 1B 4B n1 n2 data, n1 n2 is double figures in hexadecimal $n 1$ indicates that width is $n 1$ dot. Height is the eighth dot (fixed values), $n 1$ can read values from 1 to the maximum dot of each line of the printer. The printing width is $n 2 \times 256+n 1$. Because the width of most micro-printer heads is less than 256, $\mathrm{n} 2$ is 0 all the time. ESC K or 1B 4B is char printing controlled code. Data is the content of each line of bytes, Chinese characters or characters from left to right. The number of bytes should be less or equals to n1, as Figure 3 . To print special character " $\mathrm{C}$ ", its data hexadecimal is : the upper graph $20 \mathrm{H}, 50 \mathrm{H}, 88 \mathrm{H}, 88 \mathrm{H}, 50 \mathrm{H}, 27 \mathrm{H}, 08 \mathrm{H}$, 
$10 \mathrm{H}, 20 \mathrm{H}, 04 \mathrm{H}, 04 \mathrm{H}, 04 \mathrm{H}, 02 \mathrm{H}, 10 \mathrm{H}, 08 \mathrm{H}, 00 \mathrm{H}$; the lower part of graph $00 \mathrm{H}, 00 \mathrm{H}, 00 \mathrm{H}, 00 \mathrm{H}, 00 \mathrm{H}$, $\mathrm{F} 0 \mathrm{H}, 08 \mathrm{H}, 04 \mathrm{H}, 02 \mathrm{H}, 02 \mathrm{H}, 02 \mathrm{H}, 02 \mathrm{H}, 04 \mathrm{H}, 08 \mathrm{H}, 10 \mathrm{H}, 00 \mathrm{H}$.

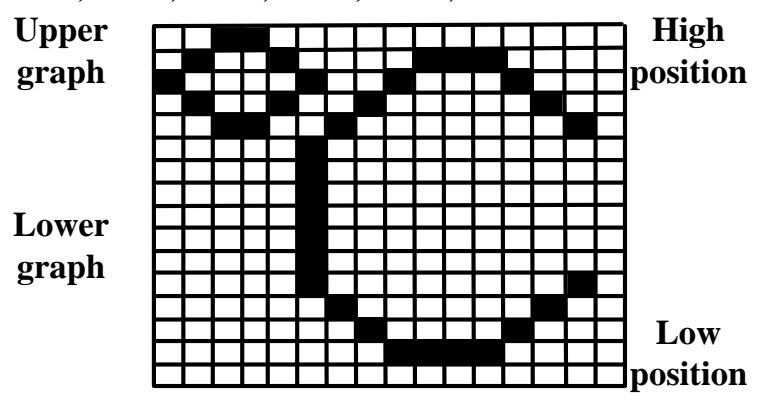

Fig.3 Dot matrix code graph

The lattice is formed according to the joint Figure of single chip with MP-T micro-printer in Figure 2 and the graph "C" in Figure 3. The program flow Figure that a single chip connects MP-T micro-printer to print special characters can be programmed, as the indication of Figure 4, Figure 5 and Figure 6. The printing task can be completed in accordance with the program flow writing.

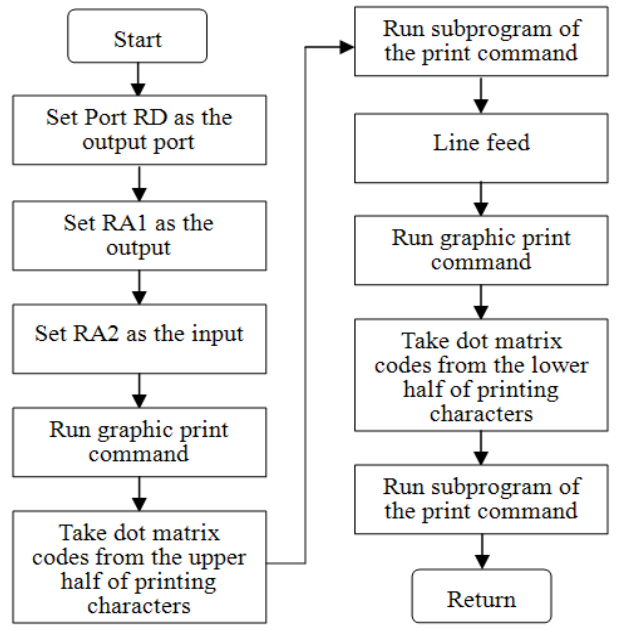

Fig.4 Main program

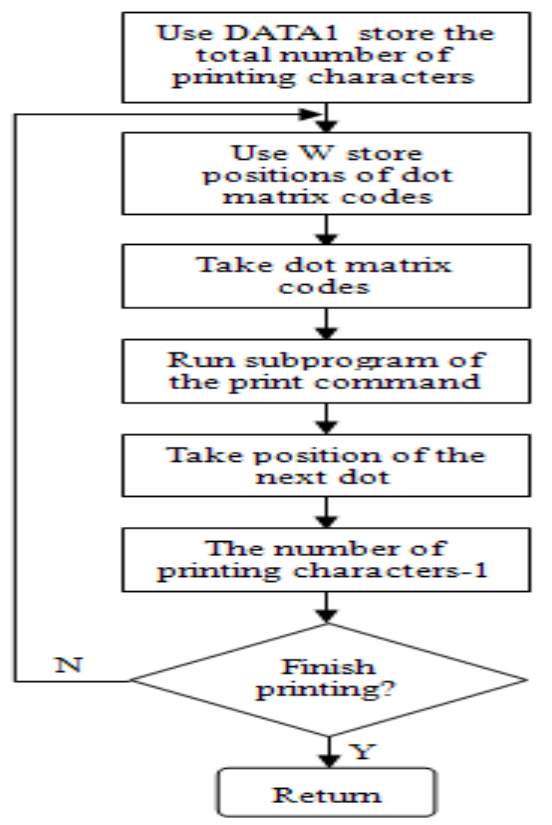

Fig.5 The print command subroutine 


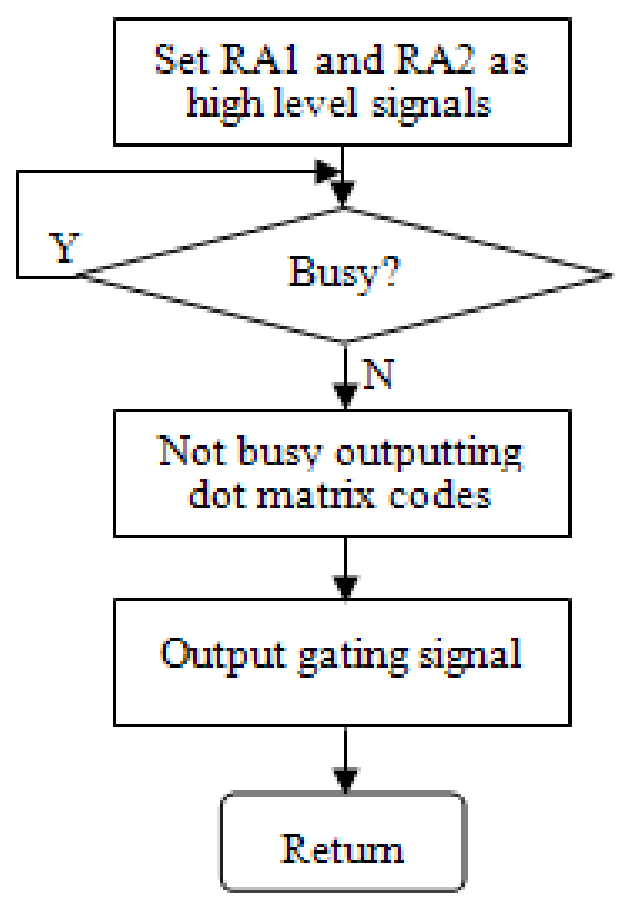

Fig.6 Dot matrix print subroutine

\section{References}

[1] Maofa Gong: Single Chip Microcomputer Interface Instance Set [M].(Beijing University of Aeronautics and Astronautics Press. Beijing China 1998.4).

[2] Zhusen Xie: $M C U$ development and typical application and design [M].(China University of Science and Technology Press. Hefei China 1997). 